\title{
How to make your competitive intelligence ventures work (3)
}

\author{
Marié-Luce Muller \\ IBIS Business and Information Services \\ mlm@ibis.co.za \\ www.ibis.co.za
}

\section{Introduction}

The previous three articles in this series focused on elements of competitive intelligence that would, if applied well, contribute to a successful and effective competitive intelligence (CI) programme in a company or organization. Various constructs or dimensions of CI were used as areas in which certain things must go right! These were mentioned briefly as being planning and focus, collection, data analysis and quality control, intelligence dissemination (communication), awareness and culture, process and structure and skills development.

$\mathrm{CI}$ is a strategic management tool. Use it as such. It is not a nice-to-have. According to Margaret Gross (Gross 2000), the ultimate objective of good CI work is the formulation of sound, fact-based, rational decisions for action. All companies, small or large, need to have some form of CI steered by a dedicated CI unit. Actionable decisions result when the intuition and background knowledge of team members couples with the computational efficiency of information technology. The last article warned that, despite having all the right ingredients, mishaps will probably still occur. These mishaps are the unfortunate results of a myriad of reasons and motives. Some are beyond the CI practitioner's control most are preventable.

In this issue, the focus is on how to best communicate intelligence to ensure actionability and, at the same time, build a competitive culture in your company. A CI programme will be rendered useless if the final message (the intelligence) gets lost in cumbersome, ill-timed, generalized reporting and communication.

\section{Art and science}

CI is often described as more art than science, a discipline that is often tacit in nature, more qualitative than quantitative in design. The true art aspect shines through when the core message of intelligence is communicated. How the intelligence is packaged (in news briefs, alerts, e-mails, SMSs, comprehensive industry estimates, in competitor profiles, etc.), when it is communicated and the content of what is communicated ultimately determines the effectiveness and value of a CI programme. Arguments should be clear and persuasive and MUST answer to intelligence needs of the programme's clients (executives, managers, etc.).

\section{The right mix}


When delivering competitive insights, timing is important. This is easier said than done. Intelligence has a limited shelf life but impact can also be lost if it is reported too early. Sometimes a CI practitioner in haste and excitement to report an ostensibly important piece of information fails to check the validity or context information. This can cause irreparable harm in terms of trust, credibility and image.

Here are a few clues as to what occurrence might turn into a reporting moment:

- A regular event along a time scale, such as the economic situation following the oncemonthly announcement of change in the cost of living index.

- A regular event along a developing occurrence, such as every time a competitor conducts a test of a newly developed technical device; fluctuation in the exchange rate of a currency.

- An interim occurrence within an intelligence collection process (usually of a complex or long-term nature) when there is a particularly large accumulation of data; a breakthrough in a particular collection effort; or a need to assess the point reached in the collection task (where do we go from here?).

- On ad hoc basis when clients require immediate intelligence input even though the particular task has yet to be completed.

- An unexpected event, a surprise of threat (early warning); a sudden opportunity when quick decisions may be required within fast reaction times, etc.

- At the conclusion of a specific key intelligence needs assignment.

\section{Mutual trust}

Delivering quality, well-timed intelligence is winning only half the battle - there is also the question of building a confidence base with the users of intelligence. This is a long-term goal. A political campaign of building trust in the intelligence is nothing more than selling ideas - this marks the difference between a smart analyst and a successful one (Sperger 2003). The intelligence that is delivered should also be focused on the users' intelligence needs (those key intelligence needs mentioned in previous articles). Competitive intelligence programmes exist to assist executives; understanding how to develop close working relationships with intelligence users is central to both the application of intelligence products and the survival of the intelligence programme.

- Make sure the recipient of your intelligence products has his other question answered!

- Make sure the answer can be used (is actionable).

- Make sure the answer is understood (Muller 2002).

\section{Other ingredients to complete the recipe}

- Report facts in a focused manner: The client wants answers to the questions regarding the following: What is known; what is unknown; what important things are unknown and what are the consequence of that?

- Base opinions on sound argument: Opinions should indicate what you know, the degree of reliability as well as what you do not know and what the consequences of that might be.

- Do report rumours and hunches: Although these soft issues are often seemingly baseless, they might have value and need to be reported. Do, however, state in the report that these are rumours that have not yet been substantiated.

- Refrain from fortune telling: Future developments can only be predicted on the basis of available evidence and expertise (forecasting). In the same vein, deductions must be the result of evidence and integration of information and not simply star gazing.

- Strive to be objective: The reporter should report what is reality and not what the 
reporter wants to see. Report things as they are and do not cloud or cushion hard facts, even though the client might not want to hear them. The objectivity required of CI professionals must be reflected in the reports that they write, even though the purpose of that report is to persuade (Ward 1999).

- Include various predictions but do indicate the most probable outcome and propose various proactive or counter strategies.

Dare to speak

Neutrality should not feature in reporting. CI practitioners are the 'chief bearers of the truth' and cannot be neutral. The truth however must be reported impartially. It is helpful to have the evidence ready to back up arguments and field questions.

\section{Clearing the myths}

A while ago, SCIP's CI Magazine published an article by Constance T. Ward in which she highlighted some myths about CI reporting (Ward 1999). These myths can briefly be summarized as follows:

- Myth: CI professionals aren't writing at all; they're just recording facts. Reality: If you express the passionate conviction of truth in your writing, you will be more persuasive.

CI professionals seek the truth in order to change their colleagues' views about the competitive situation, thus steering the company back on course or nudging it onto a new course ... the right course. The goal of a CI report should be 'to move' people to action by showing them the true picture. Ward writes: 'The CI report must clearly present the significance of the collected findings and point out the recommended path based on that intelligence.'

- Myth: CI professionals should write their reports when the data are ready. Reality: If you can't anticipate the answer, don't ask the question.

'What will you do with this information?' is the first question a CI professional should ask an internal client. Understanding the goals of the investigation and the possible political implications of positive or negative findings will assist in planning the research. It also will help researchers avoid the pitfall of gathering too much unnecessary, unfocused information that will not be used in the final report.

- Myth: Writing is easy for those who have the word 'intelligence' in their job titles. Reality: Not true, but you can improve on your skills.

The success of a CI report hangs on the quality and the character of both evidence and the writer. A polished, easily readable and pleasant looking report will enhance the message that must be conveyed.

\section{References}

Muller, M-L. 2002. Creating intelligence. The Nuts and Bolts Business Series, Knowledge Resources.

Sperger, M. 2003. Communicating Intelligence that makes a difference. scip.online, Issue 32. May.

Ward, C.T. 1999. Myths about CI report writing. CI Magazine. SCIP, 2(1) January-March. 
Marié-Luce Muller is a consulting competitive intelligence analyst with IBIS Business and Information Services (Pty) Ltd, a leading Pretoria-based CI consultancy. She has a distinguished career in competitive intelligence. Her primary experience lies in assisting companies in honing their CI capabilities. She also performs tracking and scanning activities on behalf of companies. Marié-Luce has published many articles on competitive intelligence (CEO Magazine, Finance Week, Business Week, Beeld, Die Burger, and the South African Journal of Business Management), including an article on South Africa as an emerging CI player, which was published in an international publication of the Society of Competitive Intelligence Professionals (SCIP). She has also published a series of booklets on competitive intelligence (Nuts and Bolts business series, published by Knowledge Resources) and is a member of a research team participating in an international study of competitive intelligence practices among exporting companies. Previously, she was involved in research into the status of competitive intelligence practices in South Africa. A member of SCIP, she holds a postgraduate degree from the University of Stellenbosch.

\section{Disclaimer}

Articles published in SAJIM are the opinions of the authors and do not necessarily reflect the opinion of the Editor, Board, Publisher, Webmaster or the Rand Afrikaans University. The user hereby waives any claim he/she/they may have or acquire against the publisher, its suppliers, licensees and sub licensees and indemnifies all said persons from any claims, lawsuits, proceedings, costs, special, incidental, consequential or indirect damages, including damages for loss of profits, loss of business or downtime arising out of or relating to the user's use of the Website. 